\title{
Représentations de l'étranger dans les récits de voyageurs français du XVIIe siècle
}

\author{
Olga Kulagina \\ Université pédagogique d'État de Moscou, Russie
}

\section{Introduction}

Le XVIIe siècle fut une période complexe en ce qui concerne la perception de l'étranger et de l'Autre en général par la société française de l'époque. ${ }^{1}$ D'un côté, le religieux définit par beaucoup l'état d'esprit de cette période qu'André Latreille caractérise comme celle de l'«intolérance légale» (Latreille 411) par rapport aux populations juive et protestante: on voit durcir les répressions officielles contre celles-ci (dont la révocation de l'édit de Nantes en 1685) et grandir le mécontentement des civils, car ces «exclus» avaient plus de succès en affaires, notamment en commerce, et étaient par conséquent plus aisés. De l'autre côté, les Français continuent la tradition des grandes découvertes en explorant des territoires nouveaux. Ce sont, avant tout, les missionnaires qui entreprennent des voyages vers l'étranger, notamment vers l'Est, afin d'y propager le catholicisme; toutefois, les laïcs sont également assez actifs dans ce domaine vu le développement intense des échanges commerciaux avec les pays orientaux. La plupart des voyageurs ont laissé des témoignages qui servent aujourd'hui de sources d'information précieuses permettant de mieux comprendre la vision de l'ailleurs à l'époque. Ces récits de voyage obéissaient, le plus souvent, à une tendance générale qui prescrivait non seulement une relation extrêmement détaillée de l'aventure et de l'inventaire (ce qui ne fait d'ailleurs qu'ajouter de la valeur à ces textes) mais aussi un respect quelque peu excessif du référentiel, à tel point que ce genre littéraire se fait qualifier de «palimpseste» et de «plagiat» (Moureau 18). Ces contraintes génériques étaient à la fois la cause et la conséquence de nombreuses idées reçues (plus ou moins justes) constituant la perception de l'étranger par la société française.

Nous nous proposons dans cet article de comparer les procédés d'écriture à l'œuvre dans deux approches de l'ailleurs par deux types de voyageurs français du XVIIe siècle, l'un laïc, l'autre religieux. Il s'agit de Les Six Voyages de Jean Baptiste Tavernier, écuyer baron d'Aubonne, qu'il a fait en Turquie, en Perse, et aux Indes... par Jean-Baptiste Tavernier (1676) et Voyage en divers États d'Europe et d'Asie entrepris pour découvrir un nouveau chemin à la Chine par Philippe Avril (1692). Les objectifs des deux voyageurs n'étaient pas les mêmes: Jean-Baptiste Tavernier n'avait pour tâche que des

\footnotetext{
${ }^{1}$ Nous nous référons aux définitions données par Jean-François Rey dans le Dictionnaire de l'altérité et des relations interculturelles $(4: 129-130)$ : le terme «altérité» désignerait «une qualité ou une essence, l'essence de l'être-autre», celui de «autre» servant à nommer «l'autre homme, autrui, l'Autre». La notion d' «étranger», à son tour, serait «souvent présentée comme une espèce particulière du genre de l'Autre» et recouvre les réalités ayant rapport particulièrement à ce qui vient du dehors. Les deux termes se révèlent pertinents pour notre étude, l'altérité étant « le sentiment intime d'une différence, mais dans son rapport à une identité qui s'appelle le moi: l'autre ne peut exister sans un moi qui le définit en se posant», alors que «l'étrangeté est la perception d'une césure que rien ne peut remplir, d'une présence qui n'a nul besoin de moi» (Moureau 10-11). Le matériel littéraire envisagé dans notre article, évoque les deux notions à la fois, ce qui sera mis en évidence tout au cours de l'analyse.
} 
négociations commerciales, alors que Philippe Avril était un prêtre jésuite chargé d'une double mission: l'ouverture d'une route terrestre vers la Chine, cette dernière lui étant confiée par Louis XIV, et la conversion des soi-disant «païens» (des Chinois, en particulier). Nous en voyons la preuve dans la préface de l'ouvrage: «Un des plus zelez Missionnaires de la Chine, qui a vieilly sous le faix des travaux Evangeliques me representoit il y a quelques années ... l'etat de ces Missions dans une lettre où il m'invitait à aller partager avec luy les grandes occupations ausquelles il ne peut plus suffire » (Avril 2).

\section{Représentation de l'ailleurs chez Jean-Baptiste Tavernier}

L'ouvrage en deux volumes de Jean-Baptiste Tavernier englobe plus de 40 ans de voyages et fait penser, avant tout, à un guide touristique détaillé où l'auteur a soigneusement recueilli jusqu'aux moindres nuances de la vie quotidienne et de la culture des peuples qu'il avait rencontrés au cours de ses expéditions: il s'agit, plus précisément, des Arméniens, des Turcs, des Arabes, des Persans, des Hindous. Cette approche s'inscrit parfaitement dans la tendance que nous avons évoquée plus haut, les écrivains-voyageurs visant avant tout à renseigner le lecteur et à présenter à ce dernier un «témoignage historique» aussi précis que possible (Gohard-Radenkovic 82; Mervaud et Roberti 17). Notons que Tavernier semble, à première vue, ne se soucier que peu des différences religieuses (d'ailleurs, pertinentes à l'époque) en se focalisant sur de simples plaisirs terrestres. L'analyse des exemples suivants permet de mieux illustrer cette préférence du voyageur: :

Le vin estoit excellent, \& il ne nous manqua rien selon le pays pour la bonne chere. C'est la coûtume parmi les Armeniens de presenter aux conviez un peu avant le repas une grande coupe d'eau de vie, avec des dragées de plusieurs sortes, $\&$ des écorces confites d'orange $\&$ de citron dans sept ou huit pourcelaines arranges dans un grand basin de ces laques de la Chine. C'est un petit prelude pour exciter l'appetit; les Armeniens et les femmes mesme vuident de grandes tasses d'eau de vie (Tavernier $1: 45)$

La grande quantité de pistaches qui sont de la Perse vient de Malavert, petite ville à douze lieuës d'Ispahan en tirant au levant : Ce sont les meilleures pistaches du monde \& le terroir qui est de grande étenduë en produit dans une telle abondance, qu'il y en a dequoy fournir toute la Perse \& toutes les Indes (Tavernier 1: 62)

Kom est une des grandes villes de la Perse dans un pays plat $\&$ fort abondant en riz. Il y croist aussi de bons fruits, \& particulièrement de grosses \& excellentes grenades (Tavernier 1: 67)

Le terroir de Smyrne est fertile \& abondant en toutes choses necessaires à la vie, mais particulierement en excellents vins \& en bonnes huiles. Il y a des salines à demy lieuë de la ville du côté du nord. La mer fournit quantité de bon poisson, toute sorte de chasse y est à tres grand marché; en un mot, Smyrne est une ville de bonne chere (Tavernier 1: 77)

Les énumérations, ainsi que les nominations isolées des aliments de toutes sortes (le vin, une grande coupe d'eau de vie, des dragées, des écorces confites d'orange $\&$ de citron, les pistaches, ...abondant en riz, de bons fruits, 
de grosses \& excellentes grenades, excellents vins, bonnes huiles, bon poisson) laissent entendre que l'auteur prend plaisir à ces réalités nouvelles et sait bien les apprécier. La répétition des épithètes mélioratives bon / bonne et excellent / excellente (cette dernière portant aussi une certaine valeur hyperbolique) sert de même à mettre en valeur les impressions favorables du voyageur. Cependant, en ce qui concerne les détails de la vie religieuse des étrangers, Tavernier ne manque pas non plus de sens d'observation. Ainsi, en décrivant les églises arméniennes, il relève une particularité culturelle que nous verrons plus tard figurer dans le récit de Philippe Avril:" Comme ils faisoient d'abord de grands gains, ils faisoient souvent des voeux \& donnoient beaucoup à cette Eglise, où il y a aujourd'huy d'aussi riches ornements qu'en aucune Eglise de la Chrestienté » (Tavernier 1: 31).

Nous voyons que les Arméniens, selon Tavernier, auraient tendance à mettre en valeur les apparences et l'aspect extérieur de leur pratique religieuse, ce qui est exprimé par l'hyperbole d'aussi riches ornements qu'en aucune Eglise de la Chrestienté (31). Toutefois, la richesse du décor des églises en Arménie ne semble point l'offusquer, vu l'absence de lexèmes ou de procédés à connotation tant soit peu dépréciative du contexte immédiat. Le voyageur remarque toutefois des divergences d'ordre religieux, ainsi que certaines pratiques de cette nature qu'il trouve étranges voire inadmissibles. Citons, à titre d'exemple, un passage qui illustre les relations entre les Arméniens et les Turcs:

Tout le pays qui est entrecoupé de ces rivières d'Aras \& de Kars, \& de plusieurs autres qui s'y viennent joindre, n'estant presque habité que par des Chrestiens, le peu de Mahometans qui s'y trouvent sont si superstitieux, qu'ils ne boivent point de l'eau d'aucune de ces rivieres, \& ne s'y lavent point, les tenant impures et souillées par les Chrestiens qui s'en servent. Ils ont des puits $\&$ des cisternes en leur particulier, \& ils ne souffrent pas que les Chrestiens en approchent, tant il y a de superstition \&de folie parmi les Mahometans de ces quartiers-là. Mais il n'y en a pas moins parmi les femmes Armenienes de Zulfa..., lesquelles aussi sont si scrupuleuses qu'elles ne veulent point boire de l'eau de la riviere de Senderou, qui passe à Ispahan, parce que les Mahometans s'y lavent, \& elles ne boivent que de l'eau de leurs puits, ne voulant pas mesme manger des viandes qui ont esté tuées par les Mahometans (Tavernier 1:23)

Notons que les Turcs sont désignés de manière neutre, sans aucune allusion à leur prétendue «infidélité», celle-ci étant toutefois une des caractéristiques récurrentes des Orientaux à l'époque. Il ne s'agit pas d'une antithèse «bon catholique / chrétien - idolâtre / infidèle», mais de celle «chrétien - mahométan», qui ne porte aucune nuance évaluative ou généralisante et peut donc être considérée comme assez objective.

Toujours à propos de la représentation des Turcs, signalons un autre détail de leur portrait qui n'a rien à voir avec la religion, c'est-à-dire leur prétendue avidité pécuniaire: «Ainsi le Janissaire fut puni par la partie la plus sensible parmi les Turcs qui preferent l'argent à toutes choses ... » (Tavernier 1: 85). L'hyperbole qui preferent l'argent à toutes choses produit l'effet d'une vision stéréotypée, vision qui se stabilisera parmi les voyageurs français aux 
siècles suivants. ${ }^{2}$

Enfin, la représentation des Arabes montre l'évolution de celle-ci depuis le Moyen âge. On sait que les Arabes étaient dépeints en véritables démons du combat dans les chansons de geste médiévales et que leur vaillance et leur audace étaient absolument reconnues (malgré la tonalité fort agressive de la totalité des textes) ce qui est prouvé par une grande fréquence d'épithètes évocatrices dans les textes épiques, à savoir hardiz - hardi, prouz - preux (La Chanson de Guillaume), vaillant (La Chanson de Roland), forz - fort (La Chanson de Guillaume, La Chanson de Roland), fer - farouche, redoutable (Roland à Saragosse). Au XVIIe siècle, dans le texte de Jean-Baptiste Tavernier, les Arabes perdent de leur ancienne réputation et se voient réduits à de vulgaires brigands: « ... si la Caravane faisoit mine de passer outre sans payer les droits, ces Princes Arabes la tailleroient toute en pieces, ou prendroient tous les chameaux, ou voleroient tout, comme ils ont fait quelquefois » (Tavernier 2: 3). Ce passage nous présente une antithèse sémantique Princes - voler. Le lexème prince n'est normalement pourvu que de connotations mélioratives en traduisant l'idée de noblesse et de richesse voire de splendeur, ce qui ne semble que peu compatible avec une rafle en plein jour. Pourtant, c'est par le biais de cette antithèse que Tavernier souligne la prétendue bassesse des habitudes des Arabes auxquelles même la soi-disant élite ne ferait pas exception.

Il semble également qu'au premier abord, la vision des étrangers par Jean-Baptiste Tavernier est dépourvue (ou presque) de l'influence religieuse, cependant cette impression n'est pas tout à fait fondée. En effet, Tavernier ne manque pas de mettre en relief la distinction entre les représentants des religions monothéistes, que ce soit le catholicisme ou une autre confession, et les vrais païens (ce qui était rare à l'époque, tous les non-catholiques étant classés, dans la plupart des cas, parmi les « idolâtres »): « ... hors des Gouverneurs des lieux $\&$ quelques-uns de leurs domestiques qui sont Mahometans, tous les peuples sont Idolâtres » (Tavernier 2: 164). L'antithèse Mahometans - Idolâtres démontre une certaine objectivité de l'auteur en ce qui concerne sa perception de l'altérité religieuse. Mais cette objectivité s'arrête là où il est question des soi-disant idolâtres, c'est-à-dire ceux qui appartiennent à des religions polythéistes :« On fait compte qu'il y a aux Indes jusqu'à huit cent mille Fakirs Mahometans, \& douze cens mille d'Idolâtres, ce qui est un nombre prodigieux. Ce sont tous des vagabonds $\&$ des faineans, qui ébloüissent les yeux des peuples par un faux zele, \& leur font accroire que tout ce qui sort de leur bouche est un oracle » (Tavernier 2: 365). Les lexèmes à valeur dépréciative (des vagabonds, des faineans), l'épithète faux zele, ainsi que l'hyperbole à effet ironique ... leur font accroire que tout ce qui sort de leur bouche est un oracle traduisent la perception condescendante de l'ailleurs par l'auteur, signe d'une hiérarchisation des cultures où les pratiques païennes sont jugées inférieures à celles des partisans du monothéisme. Citons encore quelques exemples accentuant l'ironie de Tavernier par rapport à ces croyances:

Bien que les Idolâtres des Indes rendent à la créature, comme à la vache,

\footnotetext{
${ }^{2}$ Voir, en guise d'exemple, Itinéraire de Paris à Jérusalem de Chateaubriand.
} 
au singe, $\&$ à divers monstres, les mémes honneurs qui ne sont dûs qu'à la véritable Divinité, pourtant il est certain qu'ils reconnoissent un seul Dieu infini, tout-puissant \& tout sage, Createur du Ciel \& de la Terre, \& qui remplit toutes choses par sa presence. Ils l'appellent en quelques endroits Permesser ${ }^{3}$, en d'autres Peremaël ${ }^{4}$ comme vers la coste des Malvares, \& Vuishnou ${ }^{5}$ dans la langue des Bramins qui habitent la coste de Coromandel. Comme ils ont peut-estre oüi dire que la figure circulaire est la plus parfaite de toutes les figures, ils ont crû raffiner en disant que Dieu est d'une figure ovale, \& c'est pour cette raison qu'ils tiennent ordinairement dans leurs Pagodes un caillou en ovale qu'ils apportent du Gange \& qu'ils adorent comme Dieu. Ils sont si fort arrestez à cette folle imagination que les plus sages d'entre les Bramins ne veulent pas méme écoûter de raisonnement contraire, $\&$ ainsi il ne faut pas s'étonner si un peuple qui a de si mauvais guides tombe dans une si grossiere \& si monstrueuse idolâtrie. Il y a une Caste qui est si superstitieuse sur cet article, que ceux qui en sont tiennent de ces pierres ovales pendües à leur cou, \& s'en donnent contre l'estomac tandis qu'ils fonr leur priere.

Dans cette crasse \& pitoyable ignorance les Idolâtres comme les anciens payens font n'aistre leur dieux des hommes, \& leur donnent méme des femmes, s'imaginant qu'ils aiment les mémes choses à quoy les hommes prennent plaisir (Tavernier 2: 373)

C'est un des articles de la créance des Idolâtres, que les ames des hommes sortant des corps après la mort sont presentées a Dieu, lequel selon la vie qu'ils ont menée leur ordonne des corps pour les habiter; de sorte qu'une méme personne renaît plusieurs fois au monde. Et dautant qu'il y a des hommes de mauvaise vie dereglez dans leurs moeurs \& plongez dans toutes sortes de vices, Dieu envoye de semblables ames aprés s'estre separées de leurs corps, dans les corps de quelques vilains animaux, comme d'ânes, de chiens, de chats \& autres pour faire penitence de leurs crimes dans ces infames prisons. Mais ils croyent que les ames qui entrent dans les corps des vaches sont bien-heureuses, parce qu'ils tiennent ces animaux pour une manière de Divinité. Un homme mourant avec une queuë de vache à la main, cela suffit, disent-ils, pour le rendre bien-heureux en l'autre vie (Tavernier 2: 380)

Ces deux exemples font voir d'une façon assez explicite la prise de position de Tavernier. Malgré de nombreux détails évoqués dans les passages ci-dessus (à commencer par les noms des divinités et à continuer avec les exemples de la pratique religieuse des Hindous), le lecteur comprend que l'auteur lui-même n'en croit pas un seul mot - effet assuré par de nombreux éléments modaux rendant le doute et la subjectivité, tels que les verbes croire, s'imaginer, tenir < ces animaux > pour < une manière de Divinité> ou l'adverbe modal peut-estre. Les épithètes métaphoriques, dont certaines portent également une valeur hyperbolique (cette folle imagination, une si grossiere \& si monstrueuse idolatrie, cette crasse \& pitoyable ignorance), servent, à leur tour, à exprimer le choc éprouvé par le voyageur français au contact avec une altérité aussi tranchante. Ce choc est accompagné d'un dégoût et d'une réaction

\footnotetext{
${ }^{3}$ Mis en italique par l'éditeur.

${ }^{4}$ Idem.

${ }^{5}$ Idem.
} 
ironique assez marquée, cette dernière étant rendue par l'antithèse vilains animaux, infames prisons - ames bien-heureuses, mais aussi et surtout par l'hyperbole un homme mourant avec une queuë de vache à la main, cela suffit, disent-ils, pour le rendre bien-heureux en l'autre vie. Cette phrase peut se comprendre comme une moquerie de l'auteur à l'égard des croyances étrangères et laisse entendre au lecteur que celles-ci ne valent pas la peine d'être prises au sérieux. Il est vrai que Tavernier porte une attention considérable sur les faits et les détails (ce qui se voit quand on lit les exposés qu'il fait des légendes hindoues) mais il est évident qu'il se focalise sur le côté extérieur de l'autre culture plutôt que sur la compréhension de la mentalité des peuples qu'il rencontre. Là encore, il s'agit d'une approche générale caractéristique de la plupart des relations de voyage à l'époque qui ramènent l'autre au même pour se l'approprier et le juger - attitude qui risque d'aller jusqu'à l'extrémité en se transformant en un «refus du voyage» (Mervaud et Roberti, 65) sur le plan de la perception et de l'évaluation.

Bien que Tavernier se révèle souvent sceptique voire caustique envers les religions païennes, il est à noter qu'il lui arrive d'observer certaines manifestations de l'altérité religieuse où l'ironie n'aurait plus sa place: il s'agit du bûcher pour les veuves, faisant partie à l'époque de la tradition funéraire hindoue. La description de ce rite montre un certain choc ressenti par le voyageur : "Mais les Idolâtres ne brûlent pas seulement les corps qui sont morts, leur cruelle superstition va plus avant, $\&$ ils brûlent aussi les corps des vivans. Ils font scrupule de tuer un serpent \& méme une punaise, \& tiennent pour une action de grand merite de faire mourir dans le feu une femme vivante avec le corps de son mari deffunt » (Tavernier 2: 383). L'intensité de la réaction $\mathrm{du}$ voyageur se voit traduite par une série d'oppositions qui structurent la description du rite : en premier lieuune sorte de double antithèse à caractère linéaire où un serpent \& méme une punaise sont opposés à une femme vivante, d'un côté, et la femme vivante l'est, à son tour, à son mari deffunt, suivie par une autre antithèse les corps qui sont morts - les corps des vivans, plus globale et ainsi plus fondamentale. Dans le même temps, l'épithète métaphorique leur cruelle superstition, et le mot superstition signalent l'insertion du commentaire, ici péjoratif, , et montrent que l'auteur, ne considère pas les traditions païennes comme un élément à part entière de la culture de l'autre.

En guise de bilan intermédiaire, nous constatons une représentation nuancée de l'ailleurs par Jean-Baptiste Tavernier. D'une part, cette représentation est assez objective - effet produit par la citation de nombreux détails de la vie quotidienne des peuples rencontrés par l'auteur. D'autre part, cette objectivité peut être sérieusement mise en doute par la présence de propos condescendants, parfois même mordants tenus par Tavernier sur les croyances et traditions des peuples polythéistes, ce qui démontre l'importance du facteur religieux pour le voyageur, même si ce facteur n'est pas explicitement mis en avant dans le texte.

\section{L'image de l'étranger et de l'ailleurs chez Philippe Avril}

Le récit Voyage en divers Etats d'Europe et d'Asie entrepris pour un découvrir un nouveau chemin à la Chine (le voyage même ayant été effectué entre 1680 et 1685) regroupe les impressions de l'auteur de ses contacts avec 
des étrangers non catholiques, à savoir des Arméniens, des Turcs, des Persans, des Arabes, des Kurdes, des Kalmouks, des Russes et des Mordves. Ce critère religieux se trouve être primordial pour le voyageur missionnaire, à tel point qu'il semble ignorer (du moins, en comparaison avec Tavernier) les particularités et les nuances du mode de vie de ceux qu'il lui arrive de rencontrer. En général, l'ouvrage de Philippe Avril offre au lecteur une représentation de l'«antimonde» oriental (Gohard-Radenkovic 90). Ce thème privilégié dans de nombreux récits de voyages de l'époque servait de « miroirrepoussoir » (Ibid) afin de créer l'image d'un Occident catholique quasi-parfait, gage de stabilité et de solidarité au sein de la société. Il est aussi important de noter que le père Avril se révèle un juge assez sévère pour un jésuite à l'égard des peuples non-chrétiens aussi bien qu'à l'égard des chrétiens non-catholiques, comme on peut le voir dans cette évocation des Arméniens: "Je crus que pour m'accommoder à la foiblesse de ce peuple, qui fait consister toute l'essence de la Religion dans ces observations extérieures, je devois me priver comme eux de tous les adoucissements que l'Eglise Catholique permet à ses enfants » (Avril 20). L'antithèse sémantique essence - observations extérieures évoque un caractère assez superficiel, selon Avril, de la pratique religieuse des Arméniens (idée déjà relevée dans l'ouvrage de Tavernier, bien que dans une tonalité plus indulgente que celle d'Avril), ces derniers lui semblant incapables de comprendre toute la profondeur de la vraie religion chrétienne (le catholicisme). En même temps, par le biais d'une métaphore (bien usée, d'ailleurs, mais ne perdant rien de son efficacité pragmatique) que l'Eglise Catholique permet à ses enfants, Avril identifie l'Église catholique à une mère indulgente et tolérante. Nous le verrons encore reprendre cette idée, toujours en abordant l'aspect religieux de son voyage.

Le portrait des Russes est quant à lui fondé sur un stéréotype qui ne faisait que se profiler à l'époque ${ }^{6}$ et qui a pris ensuite des dimensions assez importantes - à savoir l'habitude de lever le coude avec un certain excès:

C'est une coutume..., universellement établie dans le Païs où nous allons, de presenter de l'eau de vie, et plusieurs autres liqueurs encore plus fortes, à tous ceux qui viennent vous rendre visite, et l'on est si jaloux de vous voir agréer ces marques d'amitié qu'on vous donne, que le refus qu'on en fait passe pour un sanglant affront, qu'on ne manque jamais de vanger par quelque insulte facheuse (Avril 97)

Les procédés d'amplification dominent dans ces lignes. L'emploi de l'épithète généralisante universellement établie et de celle à valeur hyperbolique si jaloux fait penser à une règle qui n'aurait pas d'exceptions. En outre, les métaphores un sanglant affront et quelque insulte facheuse impliquent l'idée d'un délit voire d'un crime grave que seul le sang du coupable saurait laver, ne fût-ce qu'au sens figuré, ce qui implique le caractère outré des pratiques culturelles russes vues par l'auteur. Ces métaphores créent, en plus, un effet de grotesque, le «délit» n'étant que peu comparable au «châtiment» prévu. Cette façon de réagir renforce la vision de la Russie (ou de la Moscovie - nom historique qui servait à la désigner à l'époque) comme un pays aux traditions ancestrales très fortes, ce qu'elle était justement aux yeux des contemporains

\footnotetext{
${ }^{6}$ L'Europe occidentale ne faisait pour l'instant que découvrir la Russie, cette dernière étant vue comme un pays «difficilement accessible» (Broc 542) - non seulement au sens géographique du terme, semble-t-il.
} 
européens pour qui le voyage en Russie était une sorte de «voyage dans le passé » (Stroev 272). Philippe Avril obéit ici à la tendance générale, ce que l'on retrouve dans un autre exemple illustrant l'amour excessif que les Russes voueraient au verre et à la bouteille: « Il est vray..., que ces sortes d'honnetetez sentent un peu la Barbarie, mais comment faire avec un peuple qui se croit méprisé déslors qu'il voit qu'on se ménage, et qui d'ailleurs s'est tellement fait un principe de vertu de son intemperance ... » (Avril 97).

Nous sommes en présence de deux antithèses à la fois - celle de barbarie et honnetetez, d'une part, et celle de vertu et intemperance, d'autre part. Ces procédés servent à rendre le caractère contradictoire de la culture russe qui se présente devant le narrateur, mais aussi à créer un effet ironique qui traduit la vision de Philippe Avril et le regard qu'il porte sur une autre culture. Même si ce point de vue paraît exagéré), il sera activement partagé par les générations suivantes. $^{7}$ Notons que le voyageur avait de bonnes raisons personnelles de représenter les Russes d'une manière peu flatteuse: en effet, les autorités russes lui avaient refusé la permission de passer par la Sibérie pour aller jusqu'en Chine, sa destination finale, sans doute, par crainte qu'Avril et ses compagnons ne soient des espions cherchant à joindre et renseigner d'autres missionnaires jésuites installés en Chine (Mervaud et Roberti 27). Avril dut finalement rentrer en France sans avoir rempli la mission qui lui avait été confiée par le roi. Quoi qu'il en soit, les impressions du voyageur font parfaitement corps avec les idées en vigueur à l'époque, en renforçant l'image de l' " antimonde » que nous avons évoquée plus haut. L'image des Russes barbares, enclins à la démesure de toute sorte, demeurera longtemps assez tenace, malgré les tentatives des Russes eux-mêmes de se défendre en invoquant des stéréotypes et des préjugés propagés par des gens ignorants qui n'auraient rien vu ni compris de la culture russe durant leur séjour dans le pays (Mervaud 116-117). L'image négative de la Russie perdurera assez nettement jusqu'au début du XVIIIe siècle où Pierre Ier tentera de réhabiliter le pays aux yeux de l'Occident (Liechtenhan La progression de l'interdit 23).

L'observation du religieux tient, quant à elle, une place considérable dans la description des Russes et de leur culture. Voici ce que dit Avril à propos de la relation « catholicisme - orthodoxie »:

Il ne faut etre fort instruit du Schisme \& de la Religion des Grecs, dont les Moscovites font profession, pour savoir combien les Pretres \& les Religieux qui en sont le principal appuy, sont opposez à l'Eglise de Rome, \& combien grande est leur haine pour tous ceux qui luy sont unis. Elle va jusqu'à cet excés de fureur que quand il se trouve quelque malheureux ou lache Catholique qui par quelque intérêt temporel, ou par quelque autre consideration egalement criminelle, quitte sa veritable Mere pour se soumettre à cette cruelle maratre, on l'oblige

\footnotetext{
${ }^{7}$ Voir, en guise d'exemple, l'ouvrage La religion, les moeurs et les usages des Moscovites (133) par Jacques Jubé, abbé janséniste ayant effectué un voyage dans la Russie des années 1730, qui évoque la brutalité animale et l'intempérance des Russes. Par ailleurs, les relations de voyage européennes en général recouraient aux clichés se répétant d'un ouvrage à l'autre: ainsi, la Russie serait un pays de brutes, malpropres et enclines à toute sorte de perversions, quoique les voyageurs qui le prétendaient ne soient pas allés, dans la plupart des cas, plus loin que les lieux où ils étaient reçus par les autorités (Mervaud et Roberti 103, 106-107, 115-116, 118), ce qui limitait sensiblement leur champ de vision.
} 
indispensablement, ainsi que je l'ay veu moy-meme, avant que de la faire passer d'une communion à l'autre, de recevoir un second bapteme... (Avril 104)

Une fois de plus, c'est la représentation métaphorique du catholicisme qui se fait remarquer dans le passage cité: l'Église catholique est encore identifiée à une mère, cette fois trahie par un enfant infidèle et ingrat. L'idée de trahison est renforcée par la métaphore consideration egalement criminelle, ce qui a pour but de représenter la conversion à une autre branche du christianisme comme un acte impardonnable. Cette autre branche, à son tour, est aussi peinte par le biais d'une métaphore et est identifiée à une "maratre ", cruelle et indigne, ce qui devrait avoir pour effet l'impact immédiat et certain sur les sentiments les plus profonds de l'éventuel lecteur: normalement, quiconque trahirait sa mère mériterait d'être sévèrement blâmé, de même que, selon l'auteur, celui qui abandonnerait sa religion d'origine devrait être inévitablement châtié. Ce peu de cérémonie par rapport à l'orthodoxie s'inscrit bien dans la ligne générale des relations de la Russie avec le Saint-Siège, celuici la considérant à l'époque comme une " enfant abâtardie du christianisme » qu'il fallait corriger en lui faisant reprendre le prétendu droit chemin (Liechtenhan Les trois christianismes et la Russie 67). De leur côté, les Russes refusaient de laisser entrer les Occidentaux dans les églises orthodoxes et ne les recevaient à table qu'avec une certaine réticence (67), les deux branches se traitant mutuellement d'hérétiques sans parvenir à s'entendre jusqu'à l'arrivée de Pierre Ier qui entama le long processus de «réconciliation» et de reconnaissance réciproque. ${ }^{8}$

Avant de passer à l'étude du portrait des peuples non-chrétiens, nous nous arrêterons sur un autre cas particulier, qui pourrait être qualifié d'intermédiaire dans le contexte donné, à savoir la représentation d'un peuple qui à l'époque n'était pas encore tout à fait chrétien et qui, en même temps, n'était plus païen: il s'agit des Mordves (ou des Mordevates, comme ils sont appelés dans le texte de Philippe Avril) dont la christianisation fut un processus durable et douloureux et ne s'acheva que vers le XVIIIe siècle: « Nous fumes sensiblement touchez un jour que nous passames par un Bourg de ces infideles qu'on nomme Mordevates $^{9}$, du peu de soin que prennent les Moscovites, soit Pretres, soit Religieux, du salut de ces malheureux Idolatres, qui sont etablis en assez bon nombre dans le centre même de la Moscovie, et qu'il seroit aisé d'attirer à la connoissance du vray Dieu » (Avril 129).Les termesinfidèles et ces malheureux Idolatres attirent particulièrement notre attention, malgré leur emploi tout à fait usuel dans la littérature française de l'époque, pour désigner les représentants d'autres cultures et d'autres religions. Si le lexème infidèle peut être défini comme qui est étranger à la religion considérée comme vraie

\footnotetext{
${ }^{8}$ Voici ce qui est dit à ce sujet dans l'ouvrage Les trois christianismes et la Russie. Les voyageurs occidentaux face à l'Église orthodoxe russe (XVe-XVIIIe siècle) de FrancineDominique Liechtenhan: « Dans les premières années du règne de Pierre Ier, les étrangers eurent enfin le droit d'entrer dans les églises orthodoxes, mais ils devaient être accompagnés d'un Russe qui les empêchait de piétiner certains endroits et de toucher les objets sacrés. L'envoyé de Hanovre, Weber, fit cette expérience, mais le prêtre ne put s'empêcher de brûler de l'encens après son départ, dans l'idée de purifier l'édifice». On en déduit que les Russes étaient loin de renoncer à leur idée de la religion, qui s'avéra fort tenace malgré l'assouplissement sensible de la politique officielle concernant la question » (112).

${ }^{9}$ Mis en gras par l'éditeur.
} 
(Le Trésor de la langue française informatisé), il est donc patent que Philippe Avril ne connaît qu'une seule vraie religion (évidemment, il n'est pas le seul à $\mathrm{y}$ croire et ses jugements ne font que refléter l'opinion «officielle») et qu'il ne prend pas en compte le fait qu'à l'époque il y a déjà beaucoup de chrétiens parmi les Mordves qui ne peuvent donc plus être définis comme idolâtres. Apparemment, le voyageur ne fait aucune distinction entre les confessions monothéistes non-catholiques et les croyances polythéistes, c'est-à-dire païennes au sens propre du terme: les deux seraient blâmables, et cela au même titre. L'épithète vray (...la connoissance du vray Dieu) démontre également le caractère formel du regard que le voyageur missionnaire porte sur l'altérité qu'il perçoit plutôt comme une étrangeté ne méritant aucune considération de sa part et ne représentant rien d'autre qu'une anomalie à corriger.

Quant à la représentation des non-chrétiens, elle n'est pas moins péjorative que celle des chrétiens non-catholiques. Dans certains cas elle l'est encore plus qu'aux époques précédentes - il s'agit notamment des Arabes dont la représentation par Avril est semblable à celle que nous trouvons dans l'ouvrage de Tavernier: «...les Arabes, qui n'ont d'autre métier que celuy de voler, et d'autre revenu que le butin qu'ils enlevent ...» (Avril 24). La dégradation de l'image des Arabes est concrétisée par l'idée explicite de la prétendue malhonnêteté des non-chrétiens, cette dernière semblant être un stéréotype déjà assez fréquent au moment de la parution du Voyage... de Philippe Avril. Cette perception stéréotypée se trouve renforcée par la description des Kurdes: « Comme ces peuples errants sont aussi redoutables que les Arabes, à qui ils ne cèdent en rien de l'art de voler, on ne songea qu'à se dérober au plutôt à leur veue... » (32). Manifestement, l'auteur est en train d'ironiser en combinant les lexèmes art et voler dont les connotations sont contraires et qui, employés ensemble, frisent l'oxymore. Au final, les procédés traduisent une attitude condescendante voire légèrement méprisante à l'égard de l'ailleurs et de ses habitants.

Cette condescendance est également présente dans la désignation des Kalmouks comme « ...cette multitude prodigieuse de Vagabonds ... » (Avril, 83). La définition citée ci-dessus serait une des moins dures dont l'auteur gratifie les peuples qu'il lui arrive de contacter. L'épithète métaphorique multitude prodigieuse est ambiguë : d'une part le nombre peut inquiéter l'observateur, de l'autre il fascine également. Cette dualité, donnée comme un fait accompli, participe à la construction d'un peuple peu compréhensible, voire mystérieux, dont le voyageur ne cherche point à percer l'énigme. En revanche, les Persans sont représentés d'une façon assez catégorique, ce qui est difficilement explicable vu l'absence de conflit ouvert entre la France et la Perse à l'époque : «...ces ennemis de notre Religion et de la leur...» (50). Cette métaphore dépréciative est renforcée par une autre qui la suit: « ... cette Nation aveugle me parut d'ailleurs fort docile et tout-à-fait raisonnable, en toute autre matière qu'en ce qui regarde la Religion » (55). L'épithète métaphorique cette Nation aveugle s'oppose à l'idée même de la raison qui est exprimée dans l'exemple ci-dessus. Cette antithèse a pour effet de dévaloriser les Persans en tant que représentants d'une religion autre que celle de l'auteur, ce qui souligne, encore une fois, toute l'importance du critère religieux pour le missionnaire français.

En même temps, aussi paradoxal que ce soit, les Turcs seraient, à 
première vue, mieux appréciés par l'auteur que les Français catholiques quand il s'agit de leur zèle religieux: "...l'exactitude que fait paroitre ce peuple infidele pour la priere, m'a fait gemir bien des fois de la negligence que temoignent nos Chretiens pour un si fait exercice » (40). L'antithèse ce peuple infidele - nos Chretiens semble équilibrée par celle de l'exactitude et la negligence : en effet, les Turcs seraient plus respectueux des horaires des prières et, par conséquent, auraient mérité plus d'estime de la part d'un ecclésiastique (ce qui tranche avec l'image des Turcs avides et terre à terre, créée par Tavernier). Toutefois, l'épithète infidèle, de même que le déterminant possessif nos, révèlent au lecteur le vrai but pragmatique de cette remarque de Philippe Avril: il ne s'agit pas de valoriser les Turcs mais plutôt de reprocher aux Français catholiques leur manque de sérieux en matière de pratique religieuse et de les motiver à remédier à la situation, puisqu'un bon chrétien, selon Avril, ne devrait jamais céder en quoi que ce soit à un «infidèle».

\section{Conclusion}

Ces deux textes, comme nous avons pu le voir, reflètent les tendances majeures déterminant la spécificité du récit de voyage du XVIIe siècle, à savoir: prédominance d'un important ethnocentrisme, vision par beaucoup stéréotypée de l'altérité culturelle, grande attention prêtée aux détails de la vie quotidienne des peuples rencontrés (notamment, en cas de l'ouvrage de Tavernier). Certains points se recoupent dans les deux relations (en particulier, dans la vision des Arabes ou celle des Arméniens), mais l'approche de Jean-Baptiste Tavernier en tant que laïc est plus nuancée que celle de Philippe Avril. En effet, Tavernier distingue les confessions monothéistes des religions païennes, ce qui est négligé par Avril qui ne fait pas de distinction entre les croyances autres que catholiques en les qualifiant toutes d'idolâtres. Toutefois, Tavernier reste aussi critique par rapport aux religions polythéistes en démontrant explicitement son attitude ironique à l'égard de celles-ci. En général, les procédés d'écriture -(antithèses, hyperboles, oxymores) s'avèrent récurrents dans les deux textes et révèlent une perception oblique (ironique) de l'altérité culturelle par les deux voyageurs, qui pourrait être caractérisée comme condescendante, frôlant à certains moments le mépris, et largement déterminée par le filtre du religieux. Ce critère perdurera dans la vision de l'ailleurs et de l'altérité chez les voyageurs des siècles à venir à concurrence de la perspective sociopolitique qui se développera au cours du XVIIIe siècle.

\section{Bibliographie}

Avril, Philippe. Voyage en divers États d'Europe et d'Asie entrepris pour découvrir un nouveau chemin à la Chine. Paris: Jean Boudot, 1693. Imprimé.

Broc, Numa. « La géographie des philosophes. Géographes et voyageurs français au XVIIIe siècle. » Thèse de doctorat. Université Paul Valéry de Montpellier, 1972. Imprimé.

La Chanson de Guillaume. Publ. par Duncan MacMillan. 2 vols. Paris: Picard, 1949. Imprimé

La Chanson de Roland. Édition critique et traduction de Ian Short. Paris: Librairie Générale Française, 2008. Imprimé. 
Gohard-Radenkovic, Aline. «"L’altérité" dans les récits de voyage. Persée. L'Homme et la société, 1999. Web. 23 oct. 2016.

« Infidèle. » Le Trésor de la Langue Française informatisé. Web. 10 juil. 2016.

Jubé, Jacques. La religion, les moeurs et les usages des Moscovites. Texte présenté et annoté par Michel Mervaud. Oxford: The Voltaire Foundation at the Taylor Institution, 1992. Imprimé.

Latreille, André. Histoire du catholicisme en France sous les Rois Très Chrétiens. 3 vols. Vol.2. Paris: Spes, 1960. Imprimé.

Liechtenhan, Francine-Dominique. « La progression de l'interdit: les récits de voyage en Russie et leur critique à l'époque des Tsars ». E-periodica. Schweizerische Zeitschrift für Geschichte $=$ Revue suisse d'histoire $=$ Rivista storica svizzera, 1993. Web. 30 oct. 2016.

Liechtenhan Francine-Dominique. Les trois christianismes et la Russie: Les voyageurs occidentaux face à l'Église orthodoxe russe (XVe - XVIIIe siècle). Paris: CNRS Éditions, 2002. Imprimé.

Mervaud, Michel. « Le knout et l'honneur des Russes (à propos de deux articles de l'Encyclopédie) ». Persée. Recherches sur Diderot et sur l'Encyclopédie, 1993. Web. 07 nov. 2016.

Mervaud, Michel et Jean-Claude Roberti. Une infinie brutalité. L'image de la Russie dans la France des XVIe et XVIIe siècles. Paris: Institut d'études slaves, 1991. Imprimé.

Moureau, François. Le Théâtre des voyages. Une scénographie de l'Âge classique. Paris: Presses de l'Université Paris-Sorbonne, 2005.

Rey, Jean-François. « Altérité. » Dictionnaire de l'altérité et des relations interculturelles Sous la direction de Gilles Ferréol et Guy Jucquois. Paris: Armand Colin, 2010. 4-7. Imprimé.

---.« Étranger. » Dictionnaire de l'altérité et des relations interculturelles Sous la direction de Gilles Ferréol et Guy Jucquois. Paris: Armand Colin, 2010. 129-132. Imprimé.

Roland à Saragosse. Publ. par Mario Roques. Paris: Champion, 1956. Imprimé.

Stroev, Alexandre. « La Russie dans l'Esprit des journaux (années 17701780) ». La presse francophone et la Russie au XVIIIe s. Web. 06 nov. 2016.

Tavernier, Jean-Baptiste. Les Six Voyages de Jean Baptiste Tavernier, écuyer baron d'Aubonne, qu'il a fait en Turquie, en Perse, et aux Indes, pendant l'espace de quarante ans, \& par toutes les routes que l'on peut tenir: accompagnez d'observations particulieres sur la qualité, la religion, le gouvernement, les coûtumes \& le commerce de chaque païs; avec les figures, le poids, et la valeur des monnoyes qui y ont cours... BnF Gallica, 1676. Web. 20 juin 2016. 